\title{
Global Dynamics of an Avian Influenza A(H7N9) Epidemic Model with Latent Period and Nonlinear Recovery Rate
}

\author{
Rui Mu and Youping Yang (D) \\ School of Mathematics and Statistics, Shandong Normal University, Jinan 250014, China \\ Correspondence should be addressed to Youping Yang; yyang@sdnu.edu.cn
}

Received 18 October 2017; Accepted 22 January 2018; Published 22 February 2018

Academic Editor: Konstantin Blyuss

Copyright ( 2018 Rui Mu and Youping Yang. This is an open access article distributed under the Creative Commons Attribution License, which permits unrestricted use, distribution, and reproduction in any medium, provided the original work is properly cited.

An SEIR type of compartmental model with nonlinear incidence and recovery rates was formulated to study the combined impacts of psychological effect and available resources of public health system especially the number of hospital beds on the transmission and control of A(H7N9) virus. Global stability of the disease-free and endemic equilibria is determined by the basic reproduction number as a threshold parameter and is obtained by constructing Lyapunov function and second additive compound matrix. The results obtained reveal that psychological effect and available resources do not change the stability of the steady states but can indeed diminish the peak and the final sizes of the infected. Our studies have practical implications for the transmission and control of A(H7N9) virus.

\section{Introduction}

Avian influenza $\mathrm{A}(\mathrm{H} 7 \mathrm{~N} 9)$ is a subtype of influenza viruses that have been detected in birds and confirmed to be low pathogenic among poultry in the past [1]. Human infections by this particular $\mathrm{A}(\mathrm{H} 7 \mathrm{~N} 9)$ virus had not previously been reported until it was found in March, 2013 in China (WHO). It appears that $\mathrm{A}(\mathrm{H} 7 \mathrm{~N} 9)$ virus has become a highly pathogenic virus for human species who directly or indirectly contacts poultry carrying virus $[2,3]$. From September 1, 2016, to April 31, 2017, 643 cases of avian influenza A(H7N9) laboratory-confirmed cases have been reported in Mainland China, including 233 cases that have died (China CDC), which imposes a serious threat to public health.

There are different types of models to analyze the dynamical behavior of avian influenza virus and assess useful control measures. Iwami et al. [4] showed that when mutant avian influenza had already occurred, reducing the contact rate of susceptible with infectious humans may have a positive effect on preventing the second outbreak. Liu and Fang [5] formulated a two-host model to investigate the impact of screening and culling of infected poultry. Liu et al. [6] considered different growth laws of the avian population, to present that the necessary and sufficient condition for periodic solution existing is the Allee effect in avian population. However, most of these models ignore the latent period between infection and symptom onset in human populations, which does exist on the basis of the reported infection cases. Hence, we introduce the incubation period into our model to further study the internal transmission mechanism of $\mathrm{A}(\mathrm{H} 7 \mathrm{~N} 9)$ virus.

When a disease breaks out, people's awareness of its severity can generate a profound psychological impact on the individuals' behaviors to reduce unnecessary contact with infections [7]. Wang et al. [8] found that $77 \%$ of urban respondents in their investigation reported that they visited live markets less often after influenza $\mathrm{A}(\mathrm{H7N} 9)$ cases were first identified in China in March 2013. Wu et al. [9] showed that, in the second wave of avian influenza A(H7N9), greater worry among respondents led to changes in protective behaviors such as less visit to live poultry markets and less purchase of live poultry. To model the reduction in contacts due to the psychological effect, various incidence rates were formulated by researchers [10-13]. In this paper, we will modify these functions to investigate the psychological effect on the transmission of $\mathrm{A}(\mathrm{H} 7 \mathrm{~N} 9)$ virus. 
In previous dynamic models of avian influenza A(H7N9), one usually assumed the recovery rate as a constant, which means that the treatments were always sufficient. But in fact, hospital resources (such as doctors, drugs, hospital bed, and isolation places) are limited to public, especially when a disease breaks out [14]. According to reported cases by CDC, human infections with $\mathrm{A}(\mathrm{H} 7 \mathrm{~N} 9)$ virus and common flu virus have similarities in infected time and the early clinical manifestations; therefore, some available hospital resources have already been occupied. Hospital bed-population ratio, the number of available hospital beds per 10,000 population, is widely used by health planners as a method of estimating resource availability to the public [15]. Abdelrazec et al. [16] established a model for the transmission dynamics of dengue fever, with the recovery rate function reflected by the hospital bed-population ratio and the number of infections. They found that both the oscillations and backward bifurcation occur attributed to limited hospital resource capacity. And that the basic reproduction ratio $R_{0}$ is not enough to determine whether the disease eliminates or not. In this paper we will introduce the recovery rate function to explore the impact of available hospital resources in spreading A(H7N9) virus.

This paper is organized as follows. In Section 2, we formulate the $\mathrm{A}(\mathrm{H} 7 \mathrm{~N} 9)$ transmission model incorporating the combined impact of psychological effect and available hospital resources. The dynamical behavior of the model is investigated in Section 3, including the existence and global stability of equilibria. In Section 4, we carry out numerical simulations to verify the theoretical results, and the conclusions and discussions for further work are presented in Section 5 .

\section{Model Formulation}

Based on information reported, there is no evidence of sustained human-to-human transmission, although there have been two family clusters reported. Thus, we always assume the transmission of $\mathrm{A}(\mathrm{H} 7 \mathrm{~N} 9)$ virus is not from person to person. In our model, we divide the poultry into two subclasses: susceptible $\left(S_{p}\right)$ and infectious $\left(I_{p}\right)$, respectively, and the human population is divided into four subclasses: susceptible $\left(S_{h}\right)$, latent $\left(E_{h}\right)$, infectious $\left(I_{h}\right)$, and recovered $\left(R_{h}\right)$. Before constructing the model, we make the following assumptions.

(i) Taking into account the factors such as poultry market mobility, environment capacity, and the existing populations, the susceptible poultry is subject to the logistic growth [6]

$$
r_{p} S_{p}\left(1-\frac{S_{p}}{K_{p}}\right),
$$

where $r_{p}$ and $K_{p}$ are the intrinsic growth rate and maximal carrying capacity of the poultry.

(ii) Due to psychological effect, the infection force may decrease when the number of infectious individuals increases. Hence, we modify a nonlinear incidence rate proposed by Liu et al. [17] to describe the transmission of the virus from infected poultry to susceptible individuals, with the following form:

$$
\beta_{h} S_{h} \frac{I_{p}}{1+a I_{h}}
$$

where $\beta_{h}$ is the transmission coefficient, $\beta_{h} I_{p}$ measures the infection force of the disease, $a$ is a nonnegative constant, and $1 /\left(1+a I_{h}\right)$ measures the inhibition due to the psychological effect.

(iii) We assume that latent humans $\left(E_{h}\right)$ do not take up the hospital bed resources during the latent period and, meanwhile, consider the impact of hospital resources on the recovery rate, first proposed by Shan and Zhu [18], which can be expressed in the following formula:

$$
\mu\left(b, I_{h}\right)=\mu_{0}+b \frac{\left(\mu_{1}-\mu_{0}\right)}{b+I_{h}},
$$

with

$$
\begin{aligned}
\lim _{b \rightarrow+\infty} \mu\left(b, I_{h}\right) & =\mu_{1}, \\
\lim _{I_{h} \rightarrow 0^{+}} \mu\left(b, I_{h}\right) & =\mu_{1}, \\
\lim _{I_{h} \rightarrow+\infty} \mu\left(b, I_{h}\right) & =\mu_{0}, \\
\lim _{b \rightarrow 0^{+}} \mu\left(b, I_{h}\right) & =\mu_{0},
\end{aligned}
$$

where $\mu_{1}$ is the maximum per capita recovery rate due to the sufficient health care resources and few infectious individuals, $\mu_{0}$ is the minimum per capita recovery rate due to the basic clinical resources, and $b$ is the hospital bedpopulation ratio which is a nonnegative constant.

Due to the above assumptions, we can formulate the system as follows:

$$
\begin{aligned}
& \frac{d S_{p}}{d t}=r_{p} S_{p}\left(1-\frac{S_{p}}{K_{p}}\right)-\beta_{p} S_{p} I_{p}, \\
& \frac{d I_{p}}{d t}=\beta_{p} S_{p} I_{p}-\left(\mu_{p}+\delta_{p}\right) I_{p}, \\
& \frac{d S_{h}}{d t}=\Lambda-\beta_{h} S_{h} \frac{I_{p}}{1+a I_{h}}-\mu_{h} S_{h}, \\
& \frac{d E_{h}}{d t}=\beta_{h} S_{h} \frac{I_{p}}{1+a I_{h}}-\left(\mu_{h}+\omega_{h}\right) E_{h}, \\
& \frac{d I_{h}}{d t}=\omega_{h} E_{h}-\left(\mu_{h}+\delta_{h}\right) I_{h}-\left(\mu_{0}+b \frac{\left(\mu_{1}-\mu_{0}\right)}{b+I_{h}}\right) I_{h}, \\
& \frac{d R_{h}}{d t}=\left(\mu_{0}+b \frac{\left(\mu_{1}-\mu_{0}\right)}{b+I_{h}}\right) I_{h}-\mu_{h} R_{h} .
\end{aligned}
$$

Detailed descriptions of system parameters and their estimated values are listed in Table 1 . The variable $R_{h}$ can be decoupled from the first four equations of system. Hence, we can reduce system (5) to the following system: 
TABLE 1: Description of parameters.

\begin{tabular}{|c|c|c|c|}
\hline Parameter & Description & Value & Reference \\
\hline$r_{p}$ & Intrinsic growth rate of poultry & $5 \times 10^{-3}$ & {$[6]$} \\
\hline$K_{p}$ & Maximal carrying capacity of the poultry & 50000 & [6] \\
\hline$\beta_{p}$ & Transmission rate from infectious poultry to susceptible poultry & - & - \\
\hline$\mu_{p}$ & Natural death rate of poultry (chicken) & $1 / 5-1 / 10$ year $^{-1}$ & [21] \\
\hline$\delta_{p}$ & Disease induced death rate of poultry & $4 \times 10^{-4}$ & {$[6]$} \\
\hline$\Lambda$ & New recruitment and newborn of human & 30 & {$[6]$} \\
\hline$\beta_{h}$ & Transmission rate from infectious poultry to susceptible human & $5 \times 10^{-9}$ & Assumed \\
\hline$\mu_{h}$ & Natural death rate of human & $1 / 70$ year $^{-1}$ & Assumed \\
\hline$\delta_{h}$ & Disease induced death rate of human & 0.077 & {$[27]$} \\
\hline$\omega_{h}$ & Progression to latent rate of human & $1 / 7$ day $^{-1}$ & $\mathrm{CDC}$ \\
\hline$\mu_{0}$ & Minimum recovery rate of human & $(0.067-0.100)$ & [27] \\
\hline$\mu_{1}$ & Maximum recovery rate of human & $\left(\mu_{0}, 10\right)$ & [18] \\
\hline$b$ & Hospital bed-population ratio & $(0,20)$ & {$[18]$} \\
\hline$a$ & Psychological effect parameter & - & - \\
\hline
\end{tabular}

$$
\begin{aligned}
\frac{d S_{p}}{d t} & =r_{p} S_{p}\left(1-\frac{S_{p}}{K_{p}}\right)-\beta_{p} S_{p} I_{p}, \\
\frac{d I_{p}}{d t} & =\beta_{p} S_{p} I_{p}-\left(\mu_{p}+\delta_{p}\right) I_{p}, \\
\frac{d S_{h}}{d t} & =\Lambda-\beta_{h} S_{h} \frac{I_{p}}{1+a I_{h}}-\mu_{h} S_{h}, \\
\frac{d E_{h}}{d t} & =\beta_{h} S_{h} \frac{I_{p}}{1+a I_{h}}-\left(\mu_{h}+\omega_{h}\right) E_{h}, \\
\frac{d I_{h}}{d t} & =\omega_{h} E_{h}-\left(\mu_{h}+\delta_{h}\right) I_{h}-\left(\mu_{0}+b \frac{\left(\mu_{1}-\mu_{0}\right)}{b+I_{h}}\right) I_{h} .
\end{aligned}
$$

For system (6), we first show the following result.

Lemma 1. The set $\Gamma \doteq\left\{\left(S_{p}, I_{p}, S_{h}, E_{h}, I_{h}\right) \in R_{+}^{5}: S_{h}+E_{h}+I_{h} \leq\right.$ $\left.\Lambda / \mu_{h}\right\}$ is a positively invariant and attracting region of system (6).

Proof. For system (6) with nonnegative initial conditions, the following holds:

$$
\begin{aligned}
& \left.\frac{d S_{p}}{d t}\right|_{S_{p}=0}=0, \\
& \left.\frac{d I_{p}}{d t}\right|_{I_{p}=0}=0, \\
& \left.\frac{d S_{h}}{d t}\right|_{S_{h}=0}=\Lambda>0 ;
\end{aligned}
$$

hence, the solutions of $S_{p}, I_{p}$, and $S_{h}$ are nonnegative. Then we get

$$
\begin{aligned}
& \left.\frac{d E_{h}}{d t}\right|_{E_{h}=0}=\beta_{h} S_{h} \frac{I_{p}}{1+a I_{h}} \geq 0, \\
& \left.\frac{d I_{h}}{d t}\right|_{I_{h}=0}=\omega_{h} E_{h} \geq 0,
\end{aligned}
$$

so all solutions of system (6) are nonnegative.
Let $N_{h}=S_{h}+I_{h}+E_{h}$, and it follows that

$$
\begin{aligned}
\frac{d N_{h}}{d t} & =\Lambda-\mu_{h} N_{h}-\delta_{h} I_{h}-\left(\mu_{0}+b \frac{\left(\mu_{1}-\mu_{0}\right)}{b+I_{h}}\right) I_{h} \\
& \leq \Lambda-\mu_{h} N_{h},
\end{aligned}
$$

which implies that

$$
\lim _{t \rightarrow+\infty} N_{h}(t)=\frac{\Lambda}{\mu_{h}} .
$$

Moreover, if $N_{h}(t)>\Lambda / \mu_{h}$, we have

$$
\frac{d N_{h}}{d t} \leq \Lambda-\mu_{h} N_{h}<0 .
$$

Therefore, each solution of system (6) with nonnegative initial conditions initiating from $\Gamma$ will remain in $\Gamma$ for $t>$ 0 .

\section{Analysis of Equilibria}

3.1. Existence of Equilibria. In this section, we study the existence of equilibria of system (6) in $\Gamma$. By setting the righthand side of system (6) to zero, we obtain the following equations:

$$
\begin{array}{r}
r_{p} S_{p}\left(1-\frac{S_{p}}{K_{p}}\right)-\beta_{p} S_{p} I_{p}=0, \\
\beta_{p} S_{p} I_{p}-\left(\mu_{p}+\delta_{p}\right) I_{p}=0, \\
\Lambda-\beta_{h} S_{h} \frac{I_{p}}{1+a I_{h}}-\mu_{h} S_{h}=0, \\
\beta_{h} S_{h} \frac{I_{p}}{1+a I_{h}}-\left(\mu_{h}+\omega_{h}\right) E_{h}=0, \\
\omega_{h} E_{h}-\left(\mu_{h}+\delta_{h}\right) I_{h}-\left(\mu_{0}+b \frac{\left(\mu_{1}-\mu_{0}\right)}{b+I_{h}}\right) I_{h}=0 .
\end{array}
$$


Therefore, the coordinates of equilibria are determined by nonnegative solutions of equations (12). Simple calculation yields that system (6) always has two equilibria $E_{01}\left(0,0, \bar{S}_{h}, 0,0\right)$ and $E_{02}\left(K_{p}, 0, \bar{S}_{h}, 0,0\right)$, where $\bar{S}_{h}=\Lambda / \mu_{h}$ for all parameter values. We call $E_{01}$ and $E_{02}$ disease-free equilibria, which represent the state that there is no infection. Using the method proposed by Diekmann et al. [19] and van den Driessche and Watmough [20], the basic reproduction number $R_{0}$ of system (6), which is the dominant eigenvalue of the next-generation matrix, can be given by

$$
\begin{aligned}
& R_{0} \\
& =\rho\left(\left[\begin{array}{ccc}
\beta_{p} K_{p} & 0 & 0 \\
\beta_{h} \frac{\Lambda}{\mu_{h}} & 0 & 0 \\
0 & 0 & 0
\end{array}\right]\left[\begin{array}{ccc}
\mu_{p}+\delta_{p} & 0 & 0 \\
0 & \mu_{h}+\omega_{h} & 0 \\
0 & -\omega_{h} & \mu_{h}+\delta_{h}+\mu_{1}
\end{array}\right]^{-1}\right) \\
& =\frac{\beta_{p} K_{p}}{\mu_{p}+\delta_{p}},
\end{aligned}
$$

where $\rho$ is the spectral radius of a matrix.

Next, we discuss the endemic equilibrium denoted by $E^{*}\left(S_{p}^{*}, I_{p}^{*}, S_{h}^{*}, E_{h}^{*}, I_{h}^{*}\right)$. From a straightforward calculation of the first and second equations of (12), we have

$$
\begin{aligned}
& S_{p}^{*}=\frac{\mu_{p}+\delta_{p}}{\beta_{p}}, \\
& I_{p}^{*}=\frac{r_{p}}{\beta_{p} R_{0}}\left(R_{0}-1\right) .
\end{aligned}
$$

Obviously, if $R_{0}>1, I_{p}^{*}$ is positive. From the last three equations of (12), the coordinates of point $E^{*}$ must satisfy

$$
\begin{aligned}
& S_{h}=\frac{\Lambda-\left(\mu_{h}+\omega_{h}\right) E_{h}}{\mu_{h}}, \\
& E_{h}=\frac{\left(\mu_{h}+\delta_{h}+\mu_{0}\right) I_{h}+b\left(\left(\mu_{1}-\mu_{0}\right) /\left(b+I_{h}\right)\right) I_{h}}{\omega_{h}} .
\end{aligned}
$$

Substituting (16) into equation $d E_{h} / d t=0$, after some calculations we have the following equation of $I_{h}$ :

$$
f\left(I_{h}\right)=m_{3} I_{h}^{3}+m_{2} I_{h}^{2}+m_{1} I_{h}+m_{0}
$$

where

$$
\begin{aligned}
m_{3} & =-a d_{0} \frac{\left(\mu_{h}+\omega_{h}\right)}{\omega_{h}}<0, \\
m_{2} & =-d_{0} \beta_{h} I_{p}^{*} \frac{\left(\mu_{h}+\omega_{h}\right)}{\mu_{h} \omega_{h}}-\left(a b d_{1}+d_{0}\right) \frac{\left(\mu_{h}+\omega_{h}\right)}{\omega_{h}} \\
& <0, \\
m_{1} & =\Lambda \frac{\beta_{h}}{\mu_{h}} I_{p}^{*}-b d_{1} \beta_{h} I_{p}^{*} \frac{\left(\mu_{h}+\omega_{h}\right)}{\mu_{h} \omega_{h}}-b d_{1} \frac{\left(\mu_{h}+\omega_{h}\right)}{\omega_{h}}, \\
m_{0} & =b \beta_{h} I_{p}^{*} \frac{\Lambda}{\mu_{h}}>0 .
\end{aligned}
$$

$$
\begin{gathered}
\text { And } \begin{aligned}
d_{i}=\mu_{h}+\delta_{h}+\mu_{i}, \quad i= & 0,1, \text { and obviously } \\
f(0) & =m_{0}>0, \\
f(+\infty) & <0 .
\end{aligned}
\end{gathered}
$$

Intermediate Value Theorem indicates that there exists at least one positive root of (17). In the following, we consider all the situations.

(i) Assuming there are three real roots, Vieta Theorem indicates that

$$
\begin{array}{r}
I_{h_{1}} I_{h_{2}} I_{h_{3}}=-\frac{m_{0}}{m_{3}}>0, \\
I_{h_{1}}+I_{h_{2}}+I_{h_{3}}=-\frac{m_{2}}{m_{3}}<0 .
\end{array}
$$

From (20) we can see that there are two cases; one is that all three roots are positive and the other is one positive and two negative roots. Equation (21) indicates that at least one root should be negative; hence, in this case, equation (21) has a unique positive root.

(ii) Otherwise, suppose that there are a pair of complex roots and a positive real root, denoted by $x+y i, x-y i$, and $z$, where $x, y, z$ are real numbers. We have

$$
\begin{aligned}
f_{1}= & \left(I_{h}-(x+y i)\right)\left(I_{h}-(x-y i)\right)\left(I_{h}-z\right) \\
= & I_{h}^{3}-(2 x+z) I_{h}^{2}+\left(x^{2}+y^{2}+2 x z\right) I_{h} \\
& -z\left(x^{2}+y^{2}\right) .
\end{aligned}
$$

Comparing with (17), $-z\left(x^{2}+y^{2}\right)<0$ holds, which contradicts with $m_{0}>0$.

In summary, we can conclude that (17) only has a unique positive root denoted by $I_{h}^{*}$. Thus there is only one endemic equilibrium $E^{*}\left(S_{p}^{*}, I_{p}^{*}, S_{h}^{*}, E_{h}^{*}, I_{h}^{*}\right)$ of system (6). The results are listed in the following lemma.

Lemma 2. In system (6) two disease-free equilibria $E_{01}(0,0$, $\left.\bar{S}_{h}, 0,0\right)$ and $E_{02}\left(K_{p}, 0, \bar{S}_{h}, 0,0\right)$ exist, where $\bar{S}_{h}=\Lambda / \mu_{h}$ if $R_{0}<$ 1 and a unique endemic equilibrium $E^{*}\left(S_{p}^{*}, I_{p}^{*}, S_{h}^{*}, E_{h}^{*}, I_{h}^{*}\right)$ if $R_{0}>1$.

3.2. The Dynamical Behavior of the Poultry-Only Subsystem. In order to better discuss the full system, we first learn the poultry-only subsystem in $\Omega=\left\{\left(S_{p}, I_{p}\right) \in R_{+}^{2}: S_{p} \geq 0, I_{p} \geq\right.$ $0\}$,

$$
\begin{aligned}
& \frac{d S_{p}}{d t}=r_{p} S_{p}\left(1-\frac{S_{p}}{K_{p}}\right)-\beta_{p} S_{p} I_{p}, \\
& \frac{d I_{p}}{d t}=\beta_{p} S_{p} I_{p}-\left(\mu_{p}+\delta_{p}\right) I_{p} .
\end{aligned}
$$

Clearly, the poultry-only subsystem (23) is independent of the full system (6). From Lemma 2, we can directly obtain two disease-free equilibria of $(23)$, denoted by $P_{01}(0,0)$ and $P_{02}\left(K_{p}, 0\right)$ and a unique endemic equilibrium, denoted by $P^{*}\left(S_{p}^{*}, I_{p}^{*}\right)$ if $R_{0}>1$. 
Linearizing the subsystem (23) at the equilibria $P_{01}, P_{02}$, and $P^{*}$, respectively, we can obtain the Jacobian matrices. For $P_{01}$, the characteristic equation always has a positive root $r_{p}$. For $P_{02}$, the characteristic equation has two negative roots $\lambda_{1}=-r_{p}, \lambda_{2}=\left(\mu_{p}+\delta_{p}\right)\left(R_{0}-1\right)$ if $R_{0}<1$. Otherwise it has one positive root. If $R_{0}>1, P^{*}$ exists and the characteristic equation is $\lambda^{2}+r_{p}\left(S_{p}^{*} / K_{p}\right) \lambda+\beta_{p}^{2} S_{p}^{*} I_{p}^{*}=0$. All roots of the equation have negative real parts. Hence, we summarize the results as follows.

Lemma 3. The disease-free equilibrium $P_{01}(0,0)$ is always unstable. Further, (i) if $R_{0}<1$, the disease-free equilibrium $P_{02}\left(K_{p}, 0\right)$ is locally asymptotically stable and (ii) if $R_{0}>1$, the disease-free equilibrium $P_{02}\left(K_{p}, 0\right)$ is unstable and the endemic equilibrium $P^{*}\left(S_{p}^{*}, I_{p}^{*}\right)$ exists and is locally asymptotically stable.

The following theorem shows the global stability of the equilibria.

Theorem 4. If $R_{0}<1$, the disease-free equilibrium $P_{02}$ is globally asymptotically stable in $R_{+}^{2}$; if $R_{0}>1$ the endemic equilibrium $P^{*}$ is globally asymptotically stable in $R_{+}^{2}$.

Proof. If $R_{0}<1$, construct Lyapunov function

$$
V_{1}=K_{p}\left(\frac{S_{p}}{K_{p}}-\ln \frac{S_{p}}{K_{p}}\right)+I_{p}
$$

Calculate the derivative $V_{1}$ along subsystem (23); it yields

$$
\begin{aligned}
\left.V_{1}^{\prime}\right|_{(23)}= & K_{p}\left(\frac{S_{p}^{\prime}}{K_{p}}-\frac{S_{p}^{\prime}}{S_{p}}\right)+I_{p}^{\prime} \\
= & r_{p}\left(1-\frac{S_{p}}{K_{p}}\right) S_{p}-\beta_{p} S_{p} I_{p} \\
& -r_{p}\left(1-\frac{S_{p}}{K_{p}}\right) K_{p}+\beta_{p} I_{p} K_{p}+\beta_{p} S_{p} I_{p} \\
& -\left(\mu_{p}+\delta_{p}\right) I_{p} \\
= & -\frac{r_{p}}{K_{p}}\left(S_{p}-K_{p}\right)^{2}+I_{p}\left(\mu_{p}+\delta_{p}\right)\left(R_{0}-1\right)
\end{aligned}
$$$$
\leq 0
$$

The set $V_{1}^{\prime}=0$ has a unique point $P_{02}$. According to the invariance principle of Lasalle, all solutions of subsystem (23) approach the largest positively invariant subset of the set $V_{1}^{\prime}=$ 0 . Hence, if $R_{0}<1, P_{02}$ is globally asymptotically stable in $R_{+}^{2}$.

If $R_{0}>1$, consider the Lyapunov function

$$
V_{2}=S_{p}^{*}\left(\frac{S_{p}}{S_{p}^{*}}-\ln \frac{S_{p}}{S_{p}^{*}}\right)+I_{p}^{*}\left(\frac{I_{p}}{I_{p}^{*}}-\ln \frac{I_{p}}{I_{p}^{*}}\right)
$$

in $R_{+}^{2}$. Calculate the derivative $V_{2}$ along subsystem (23); it satisfies

$$
\begin{aligned}
\left.V_{2}^{\prime}\right|_{(23)}= & S_{p}^{*}\left(\frac{S_{p}^{\prime}}{S_{p}^{*}}-\frac{S_{p}^{\prime}}{S_{p}}\right)+I_{p}^{*}\left(\frac{I_{p}^{\prime}}{I_{p}^{*}}-\frac{I_{p}^{\prime}}{I_{p}}\right) \\
= & r_{p}\left(1-\frac{S_{p}}{K_{p}}\right) S_{p}-r_{p}\left(1-\frac{S_{p}}{K_{p}}\right) S_{p}^{*} \\
& -r_{p}\left(1-\frac{S_{p}^{*}}{K_{p}}\right) S_{p}+r_{p}\left(1-\frac{S_{p}^{*}}{K_{p}}\right) S_{p}^{*} \\
= & -\frac{r_{p}}{K_{p}}\left(S_{p}^{*}-S_{p}\right)^{2} \leq 0 .
\end{aligned}
$$

The set $V_{2}^{\prime}=0$ has a unique point $P^{*}$. According to the invariance principle of Lasalle, all solutions of subsystem (23) approach the largest positively invariant subset of the set $V_{2}^{\prime}=$ 0 . Hence, if $R_{0}>1, P^{*}$ is globally asymptotically stable in $R_{+}^{2}$.

3.3. The Dynamical Behavior of System (6). In this section, we will discuss the dynamical behavior of system (6) and study the local stability of equilibria $E_{01}, E_{02}$, and $E^{*}$. First, we present the following results.

Lemma 5. The disease-free equilibrium $E_{01}\left(0,0, \bar{S}_{h}, 0,0\right)$ is always unstable. Further, (i) if $R_{0}<1$, the disease-free equilibrium $E_{02}\left(K_{p}, 0, \bar{S}_{h}, 0,0\right)$ is locally asymptotically stable and (ii) if $R_{0}>1$, the disease-free equilibrium $E_{02}\left(K_{p}, 0, \bar{S}_{h}, 0,0\right)$ is unstable and the endemic equilibrium $E^{*}\left(S_{p}^{*}, I_{p}^{*}, S_{h}^{*}, E_{h}^{*}, I_{h}^{*}\right)$ exists and is locally asymptotically stable.

Proof. (i) The Jacobian matrix at $E_{01}$ is

$$
\begin{aligned}
& \mathbf{J}\left(E_{01}\right) \\
& =\left[\begin{array}{ccccc}
r_{p} & 0 & 0 & 0 & 0 \\
0 & -\left(\mu_{p}+\delta_{p}\right) & 0 & 0 & 0 \\
0 & -\beta_{h} \frac{\Lambda}{\mu_{h}} & -\mu_{h} & 0 & 0 \\
0 & \beta_{h} \frac{\Lambda}{\mu_{h}} & 0 & -\left(\mu_{h}+\omega_{h}\right) & 0 \\
0 & 0 & 0 & \omega_{h} & -d_{1}
\end{array}\right] .
\end{aligned}
$$

Since the characteristic equation always has a positive root $\lambda=r_{p}, E_{01}$ is always unstable.

(ii) The Jacobian matrix at $E_{02}$ is

$$
\begin{aligned}
& \mathbf{J}\left(E_{02}\right) \\
& =\left[\begin{array}{ccccc}
-r_{p} & -\beta_{p} K_{p} & 0 & 0 & 0 \\
0 & \beta_{p} K_{p}-\left(\mu_{p}+\delta_{p}\right) & 0 & 0 & 0 \\
0 & -\beta_{h} \frac{\Lambda}{\mu_{h}} & -\mu_{h} & 0 & 0 \\
0 & \beta_{h} \frac{\Lambda}{\mu_{h}} & 0 & -\left(\mu_{h}+\omega_{h}\right) & 0 \\
0 & 0 & 0 & \omega_{h} & -d_{1}
\end{array}\right] .
\end{aligned}
$$

One root of the characteristic equation is $\lambda=\left(\mu_{p}+\delta_{p}\right)\left(R_{0}-\right.$ 1 ), and others are negative roots. Obviously if $R_{0}<1$, disease-free equilibrium $E_{02}$ is locally asymptotically stable; otherwise $E_{02}$ is unstable. 
(iii) The Jacobian matrix at $E^{*}$ is

$$
\mathbf{J}\left(E^{*}\right)=\left[\begin{array}{ccccc}
-r_{p} \frac{S_{p}^{*}}{K_{p}} & -\beta_{p} S_{p}^{*} & 0 & 0 & 0 \\
\beta_{p} I_{p}^{*} & 0 & 0 & 0 & 0 \\
0 & -\beta_{h} \frac{S_{h}^{*}}{1+a I_{h}^{*}} & -I_{p}^{*} \frac{\beta_{h}}{1+a I_{h}^{*}}-\mu_{h} & 0 & a \beta_{h} I_{p}^{*} \frac{S_{h}^{*}}{\left(1+a I_{h}^{*}\right)^{2}} \\
0 & \beta_{h} \frac{S_{h}^{*}}{1+a I_{h}^{*}} & \beta_{h} \frac{I_{p}^{*}}{1+a I_{h}^{*}} & -\left(\mu_{h}+\omega_{h}\right) & -a \beta_{h} I_{p}^{*} \frac{S_{h}^{*}}{\left(1+a I_{h}^{*}\right)^{2}} \\
0 & 0 & 0 & \omega_{h} & -d_{0}-b^{2} \frac{\left(\mu_{1}-\mu_{0}\right)}{\left(b+I_{h}^{*}\right)^{2}}
\end{array}\right] .
$$

The characteristic equation reads

$w(\lambda)$

$$
\begin{aligned}
& =\left(\lambda^{2}+r_{p} \frac{S_{p}^{*}}{K_{p}} \lambda+\beta_{p}^{2} S_{p}^{*} I_{p}^{*}\right)\left(\lambda^{3}+\eta_{2} \lambda^{2}+\eta_{1} \lambda+\eta_{0}\right) \\
& =0
\end{aligned}
$$

where

$$
\eta_{2}=\beta_{h} \frac{I_{p}^{*}}{1+a I_{h}^{*}}+2 \mu_{h}+\omega_{h}+d_{0}+b^{2} \frac{\left(\mu_{1}-\mu_{0}\right)}{\left(b+I_{h}^{*}\right)^{2}}>0
$$

$\eta_{1}$

$$
\begin{aligned}
= & \left(\beta_{h} \frac{I_{p}^{*}}{1+a I_{h}^{*}}+\mu_{h}+d_{0}+b^{2} \frac{\left(\mu_{1}-\mu_{0}\right)}{\left(b+I_{h}^{*}\right)^{2}}\right)\left(\mu_{h}+\omega_{h}\right) \\
& +\left(\mu_{h}+\beta_{h} \frac{I_{p}^{*}}{1+a I_{h}^{*}}\right)\left(d_{0}+b^{2} \frac{\left(\mu_{1}-\mu_{0}\right)}{\left(b+I_{h}^{*}\right)^{2}}\right) \\
& +a \omega_{h} \beta_{h} \frac{S_{h}^{*} I_{p}^{*}}{\left(1+a I_{h}^{*}\right)^{2}}>0,
\end{aligned}
$$

$$
\begin{aligned}
& \eta_{0} \\
& =\left(\mu_{h}+\beta_{h} \frac{I_{p}^{*}}{1+a I_{h}^{*}}\right)\left(\mu_{h}+\omega_{h}\right)\left(d_{0}+b^{2} \frac{\left(\mu_{1}-\mu_{0}\right)}{\left(b+I_{h}^{*}\right)^{2}}\right) \\
& \quad+a \mu_{h} \omega_{h} \beta_{h} \frac{S_{h}^{*} I_{p}^{*}}{\left(1+a I_{h}^{*}\right)^{2}}>0 .
\end{aligned}
$$

It follows that

$$
\begin{aligned}
& \eta_{2} \eta_{1}-\eta_{0}=\left[\left(\beta_{h} \frac{I_{p}^{*}}{1+a I_{h}^{*}}+\mu_{h}\right)+\mu_{h}+\cdots\right] \\
& \cdot\left[\left(d_{0}+b^{2} \frac{\left(\mu_{1}-\mu_{0}\right)}{\left(b+I_{h}^{*}\right)^{2}}\right)\left(\mu_{h}+\omega_{h}\right)\right. \\
& \left.+a \omega_{h} \beta_{h} \frac{S_{h}^{*} I_{p}^{*}}{\left(1+a I_{h}^{*}\right)^{2}}+\cdots\right]-\left(\mu_{h}+\beta_{h} \frac{I_{p}^{*}}{1+a I_{h}^{*}}\right) \\
& \cdot\left(\mu_{h}+\omega_{h}\right)\left(d_{0}+b^{2} \frac{\left(\mu_{1}-\mu_{0}\right)}{\left(b+I_{h}^{*}\right)^{2}}\right)-a \mu_{h} \omega_{h} \beta_{h} \\
& \cdot \frac{S_{h}^{*} I_{p}^{*}}{\left(1+a I_{h}^{*}\right)^{2}}>0 .
\end{aligned}
$$

By the Routh-Hurwitz criterion, the roots of (31) have negative real parts. The next theorem shows the global dynamics of the system.

Theorem 6. If $R_{0}<1$, the disease-free equilibrium $E_{02}$ is globally asymptotically stable in $\Gamma$; if $R_{0}>1$ and $b \geq 2 \Lambda\left(\mu_{1}-\right.$ $\left.\mu_{0}-\left(\mu_{h} / 2\right)\right) / \mu_{h}^{2}>0$, the endemic equilibrium $E^{*}$ is globally asymptotically stable in $\Gamma$.

Proof. If $R_{0}<1$, Theorem 4 indicates that the diseasefree equilibrium $P_{02}\left(K_{p}, 0\right)$ is globally asymptotically stable in subsystem (23). By calculation, system (6) can be reduced to the following system:

$$
\begin{aligned}
& \frac{d S_{h}}{d t}=\Lambda-\mu_{h} S_{h}, \\
& \frac{d E_{h}}{d t}=-\left(\mu_{h}+\omega_{h}\right) E_{h}, \\
& \frac{d I_{h}}{d t}=\omega_{h} E_{h}-\left(\mu_{h}+\delta_{h}\right) I_{h}-\left(\mu_{0}+b \frac{\left(\mu_{1}-\mu_{0}\right)}{b+I_{h}}\right) I_{h} .
\end{aligned}
$$


From the first two equations of system (34), we can obtain

$$
\begin{aligned}
& S_{h}(t)=\left(S_{h}(0)-\frac{\Lambda}{\mu_{h}}\right) \exp \left(-\mu_{h} t\right)+\frac{\Lambda}{\mu_{h}}, \\
& E_{h}(t)=E_{h}(0) \exp \left(-\left(\mu_{h}+\omega_{h}\right) t\right) .
\end{aligned}
$$

Clearly, we have that

$$
\begin{aligned}
& \lim _{t \rightarrow+\infty} S_{h}=\bar{S}_{h}, \\
& \lim _{t \rightarrow+\infty} E_{h}=0,
\end{aligned}
$$

further, since

$$
\frac{d I_{h}}{d t}=-d_{0} I_{h}-\frac{b\left(\mu_{1}-\mu_{0}\right) I_{h}}{\left(b+I_{h}\right)}<0 ;
$$

that is, $I_{h}(t)$ is a monotonically decreasing function and $\lim _{t \rightarrow+\infty} I_{h}=0$. In summary, the disease-free equilibrium $E_{02}$ is globally asymptotically stable.

If $R_{0}>1$, Theorem 4 indicates that the endemic equilibrium $P^{*}\left(S_{p}^{*}, I_{p}^{*}\right)$ is globally asymptotically stable in subsystem (23). Similarly, we can also simplify system (6) as

$$
\begin{aligned}
& \frac{d S_{h}}{d t}=\Lambda-\beta_{h} S_{h} \frac{I_{p}^{*}}{1+a I_{h}}-\mu_{h} S_{h}, \\
& \frac{d E_{h}}{d t}=\beta_{h} S_{h} \frac{I_{p}^{*}}{1+a I_{h}}-\left(\mu_{h}+\omega_{h}\right) E_{h}, \\
& \frac{d I_{h}}{d t}=\omega_{h} E_{h}-\left(\mu_{h}+\delta_{h}\right) I_{h}-\left(\mu_{0}+b \frac{\left(\mu_{1}-\mu_{0}\right)}{b+I_{h}}\right) I_{h} .
\end{aligned}
$$

From the Jacobian matrix of system (38), we can obtain the second additive compound matrix $\mathbf{J}^{[2]}$ :

$$
\begin{aligned}
& \mathbf{J}^{[2]} \\
& =\left[\begin{array}{ccc}
-j_{11} & -a \beta_{h} S_{h} \frac{I_{p}^{*}}{\left(1+a I_{h}\right)^{2}} & -a \beta_{h} S_{h} \frac{I_{p}^{*}}{\left(1+a I_{h}\right)^{2}} \\
\omega_{h} & -j_{22} & 0 \\
0 & \beta_{h} \frac{I_{p}^{*}}{1+a I_{h}} & -j_{33}
\end{array}\right] \text {, }
\end{aligned}
$$

where

$$
\begin{aligned}
& j_{11}=\beta_{h} \frac{I_{p}^{*}}{1+a I_{h}}+2 \mu_{h}+\omega_{h}, \\
& j_{22}=\beta_{h} \frac{I_{p}^{*}}{1+a I_{h}}+\mu_{h}+d_{0}+b^{2} \frac{\left(\mu_{1}-\mu_{0}\right)}{\left(b+I_{h}\right)^{2}}, \\
& j_{33}=\mu_{h}+\omega_{h}+d_{0}+b^{2} \frac{\left(\mu_{1}-\mu_{0}\right)}{\left(b+I_{h}\right)^{2}} .
\end{aligned}
$$

We choose the matrix $\mathbf{P}\left(S_{h}, E_{h}, I_{h}\right)=\operatorname{diag}\left(1, E_{h} / I_{h}, E_{h} / I_{h}\right)$ and calculate $\mathbf{P}_{f}$, which denotes the matrix whose components are $\mathbf{P}_{f_{i j}}(x)=\left(\partial \mathbf{P}_{i j}(x) / \partial x\right)^{T} \cdot f(x)$, so $\mathbf{P}_{f} \mathbf{P}^{-1}=$ $\operatorname{diag}\left(0, E_{h}^{\prime} / E_{h}-I_{h}^{\prime} / I_{h}, E_{h}^{\prime} / E_{h}-I_{h}^{\prime} / I_{h}\right)$. Rewrite the matrix $\mathbf{B}=$ $\mathbf{P}_{f} \mathbf{P}^{-1}+\mathbf{P J}^{[2]} \mathbf{P}^{-1}$ in block matrix

$$
\mathbf{B}=\left[\begin{array}{ll}
\mathbf{B}_{11} & \mathbf{B}_{12} \\
\mathbf{B}_{21} & \mathbf{B}_{22}
\end{array}\right],
$$

where

$$
\begin{aligned}
\mathbf{B}_{11} & =-j_{11}, \\
\mathbf{B}_{12} & \\
& =\left[-a \beta_{h} S_{h} \frac{I_{p}^{*}}{\left(1+a I_{h}\right)^{2}} \frac{I_{h}}{E_{h}},-a \beta_{h} S_{h} \frac{I_{p}^{*}}{\left(1+a I_{h}\right)^{2}} \frac{I_{h}}{E_{h}}\right], \\
\mathbf{B}_{21} & =\left[\omega_{h} \frac{E_{h}}{I_{h}}, 0\right]^{T}, \\
\mathbf{B}_{22} & =\left[\begin{array}{cc}
-j_{22}+\frac{E_{h}^{\prime}}{E_{h}}-\frac{I_{h}^{\prime}}{I_{h}} & 0 \\
\beta_{p}^{*} & -j_{33}+\frac{E_{h}^{\prime}}{E_{h}}-\frac{I_{h}^{\prime}}{I_{h}}
\end{array}\right] .
\end{aligned}
$$

We consider the norm $\|\cdot\|$ in $R_{3}^{+}$as

$$
\left\|\left(S_{h}, E_{h}, I_{h}\right)\right\|=\max \left\{\left|S_{h}\right|,\left|E_{h}\right|+\left|I_{h}\right|\right\},
$$

with vector $\left(S_{h}, E_{h}, I_{h}\right)$ in $R_{3}^{+}$and denote by $\mu(\mathbf{B})$ the Lozinskil measure with respect to this norm. It follows that

$$
\begin{aligned}
\mu(\mathbf{B}) & \leq \sup \left\{g_{1}, g_{2}\right\} \\
& \equiv \sup \left\{\mu_{1}\left(\mathbf{B}_{11}\right)+\left|\mathbf{B}_{12}\right|,\left|\mathbf{B}_{21}\right|+\mu_{1}\left(\mathbf{B}_{22}\right)\right\},
\end{aligned}
$$

where $\left|\mathbf{B}_{12}\right|,\left|\mathbf{B}_{21}\right|$ are matrix norms with respect to the $L^{1}$ vector norm and $\mu_{1}$ denotes the Lozinskil measure with respect to the $L^{1}$ norm.

We calculate $g_{1}=\mu_{1}\left(\mathbf{B}_{11}\right)+\left|\mathbf{B}_{12}\right|$, where

$$
\begin{aligned}
\mu_{1}\left(\mathbf{B}_{11}\right) & =-j_{11}, \\
\left|\mathbf{B}_{12}\right| & =a \beta_{h} S_{h} \frac{I_{p}^{*}}{\left(1+a I_{h}\right)^{2}} \frac{I_{h}}{E_{h}} .
\end{aligned}
$$

Hence,

$$
\begin{aligned}
g_{1} & =-j_{11}+a \beta_{h} S_{h} \frac{I_{p}^{*}}{\left(1+a I_{h}\right)^{2}} \frac{I_{h}}{E_{h}} \\
& =-\beta_{h} \frac{I_{p}^{*}}{1+a I_{h}}-2 \mu_{h}-\omega_{h}+a \beta_{h} S_{h} \frac{I_{p}^{*}}{\left(1+a I_{h}\right)^{2}} \frac{I_{h}}{E_{h}} .
\end{aligned}
$$


Further, $g_{2}=\left|\mathbf{B}_{21}\right|+\mu_{1}\left(\mathbf{B}_{22}\right)$, where

$$
\begin{gathered}
\mu_{1}\left(\mathbf{B}_{22}\right)=\max \left\{-j_{22}+\frac{E_{h}^{\prime}}{E_{h}}-\frac{I_{h}^{\prime}}{I_{h}}+\beta_{h} \frac{I_{p}^{*}}{1+a I_{h}},-j_{33}\right. \\
\left.+\frac{E_{h}^{\prime}}{E_{h}}-\frac{I_{h}^{\prime}}{I_{h}}\right\}=\max \left\{-\mu_{h}-d_{0}-b^{2} \frac{\left(\mu_{1}-\mu_{0}\right)}{\left(b+I_{h}\right)^{2}}\right. \\
+\frac{E_{h}^{\prime}}{E_{h}}-\frac{I_{h}^{\prime}}{I_{h}},-\mu_{h}-\omega_{h}-d_{0}-b^{2} \frac{\left(\mu_{1}-\mu_{0}\right)}{\left(b+I_{h}\right)^{2}}+\frac{E_{h}^{\prime}}{E_{h}} \\
\left.-\frac{I_{h}^{\prime}}{I_{h}}\right\}=-\mu_{h}-d_{0}-b^{2} \frac{\left(\mu_{1}-\mu_{0}\right)}{\left(b+I_{h}\right)^{2}}+\frac{E_{h}^{\prime}}{E_{h}}-\frac{I_{h}^{\prime}}{I_{h}},
\end{gathered}
$$

and, hence,

$$
g_{2}=-\mu_{h}-d_{0}-b^{2} \frac{\left(\mu_{1}-\mu_{0}\right)}{\left(b+I_{h}\right)^{2}}+\frac{E_{h}^{\prime}}{E_{h}}-\frac{I_{h}^{\prime}}{I_{h}}+\omega_{h} \frac{E_{h}}{I_{h}} .
$$

From the last two equations of system (38), we have

$$
\begin{array}{r}
\frac{I_{h}^{\prime}}{I_{h}}+d_{0}-\omega_{h} \frac{E_{h}}{I_{h}}=-b \frac{\left(\mu_{1}-\mu_{0}\right)}{\left(b+I_{h}\right)}, \\
\frac{E_{h}^{\prime}}{E_{h}}-\beta_{h} \frac{S_{h}}{E_{h}} \frac{I_{p}^{*}}{1+a I_{h}}=-\left(\omega_{h}+\mu_{h}\right) .
\end{array}
$$

Taking into consideration (49), the following holds:

$$
\begin{aligned}
& g_{1}=\frac{E_{h}^{\prime}}{E_{h}}-\mu_{h}-\beta_{h} \frac{I_{p}^{*}}{1+a I_{h}}-\beta_{h} \frac{I_{p}^{*}}{\left(1+a I_{h}\right)^{2}} \frac{S_{h}}{E_{h}}, \\
& g_{2}=\frac{E_{h}^{\prime}}{E_{h}}-\mu_{h}+b \frac{\left(\mu_{1}-\mu_{0}\right)}{\left(b+I_{h}\right)^{2}} I_{h},
\end{aligned}
$$

and thus

$$
\begin{aligned}
\mu(\mathbf{B}) & \leq \sup \left\{g_{1}, g_{2}\right\} \leq \frac{E_{h}^{\prime}}{E_{h}}-\mu_{h}+b \frac{\left(\mu_{1}-\mu_{0}\right)}{\left(b+I_{h}\right)^{2}} I_{h} \\
& \leq \frac{E_{h}^{\prime}}{E_{h}}-\mu_{h}+I_{h} \frac{\left(\mu_{1}-\mu_{0}\right)}{\left(b+I_{h}\right)} .
\end{aligned}
$$

We assume $b \geq 2 \Lambda\left(\mu_{1}-\mu_{0}-\mu_{h} / 2\right) / \mu_{h}^{2}>0$; hence

$$
\mu(\mathbf{B}) \leq \frac{E_{h}^{\prime}}{E_{h}}-\frac{\mu_{h}}{2},
$$

and then

$$
\begin{aligned}
q & =\lim _{t \rightarrow+\infty} \sup _{x \in \Gamma} \sup _{x \in \Gamma} \frac{1}{t} \int_{0}^{t} \mu(\mathbf{B}) d s \\
& \leq \frac{1}{t} \int_{0}^{t^{*}} \mu(\mathbf{B}) d s+\frac{1}{t} \ln \frac{E_{h}(t)}{E_{h}\left(t^{*}\right)}-\frac{\mu_{h}}{2} \frac{t-t^{*}}{t}<0 .
\end{aligned}
$$

The Bendixson condition is satisfied; then the result follows.

\section{Numerical Simulations}

In this section, we carry out numerical simulations for system (5) in order to illustrate the influences of the basic reproduction number $R_{0}$, psychological effect, and hospital resources on the disease evolution. The lifespan of poultry for chickens is 5 to 10 years under favorable conditions [21]; thus we assume the poultry can survive 8 years and fix the parameter $\mu_{p}=3.4246 * 10^{-4}$. People can usually live for 70 years, so the natural death rate of human $\mu_{h}$ is $3.91 * 10^{-5}$. The latent period is about 7 days (China CDC) and $\omega_{h}=1 / 7$. We assume the following parameters: $r_{p}=5 * 10^{-3}, K_{p}=5 * 10^{4}, \delta_{p}=4 * 10^{-4}, \Lambda=$ $30, \beta_{h}=7 * 10^{-9}, \mu_{0}=0.067, \delta_{h}=0.077$. We choose the initial values as $\left(S_{p}(0), I_{p}(0), S_{h}(0), E_{h}(0), I_{h}(0), R_{h}(0)\right)=$ $(1000000,500,100000,3,1,0)$.

Our theoretical results show that the basic reproduction number $R_{0}$ determines the global dynamics of the system (5). Fix $a=0.001, b=0.05$, and $\mu_{1}=0.1$, for $R_{0}=\beta_{p} K_{p} /\left(\mu_{p}+\delta_{p}\right)$; when $R_{0}$ equals 1 , we obtain $\beta_{p}^{*}=1.48 * 10^{-8}$. If $\beta_{p}<\beta_{p}^{*}\left(R_{0}<\right.$ 1 ), the solutions of $I_{h}$ converge to the disease-free steady state and the disease will finally be extinct (see Figure 1(a)). If $\beta_{p}>\beta_{p}^{*}\left(R_{0}>1\right)$, the solutions of $I_{h}$ converge to the endemic state, which implies that the disease will persist (see Figure 1(b)).

We then use Latin hypercube sampling (LHS) [22] and partial rank correlation coefficients (PRCCs) [23] to explore parameter space and find to which parameter the prevalence at endemic equilibrium is sensitive when parameters vary. Due to limited data on the distribution for each parameter, we choose a uniform distribution for all input parameters with the mean value listed in Table 1. PRCC results in Figure 2(a) indicate that the first four parameters with the most significant impact on the equilibrium prevalence are the psychological effect parameter $a$, the hospital bed-population ratio $b$, the minimum recovery rate of human $\mu_{0}$, and maximum recovery rate of human $\mu_{1}$. It is reasonable that the four parameters play important roles in the infections. In fact, a larger psychological effect parameter $a$ means that the public improve their awareness of $\mathrm{A}(\mathrm{H} 7 \mathrm{~N} 9)$ virus and take more preventive measures, which leads to lower incidence rate and then lower new infections. A larger hospital bed ratio $b$ indicates that more sufficient hospital resources and treatments are provided, which then can improve the recovery rate and lead to lower new infections. The results can be seen explicitly from Figure 2(b). When the impact of psychological effect and hospital resources is introduced, the amount of equilibrium prevalence obviously decreases with the parameters $a$ and (or) $b$ increasing.

To further examine the impact of psychological effect and hospital resources on infections, respectively, we take $\beta_{p}=3.5 * 10^{-8}\left(R_{0}=2.3570>1\right)$ and $\mu_{1}=0.24$ with one of parameters $a$ and $b$ fixed and the other varying. Figure 3(a) shows that slightly increasing parameter $a$ can not only diminish the final size of the infected but also result in a much lower peak of the disease. Similar results can be obtained when parameter $b$ varies (see Figure 3(b)). 


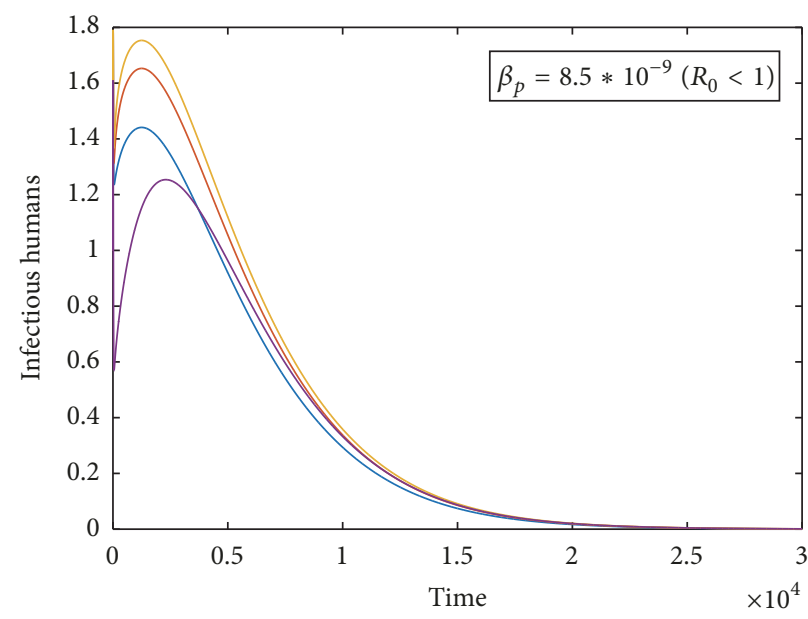

(a)

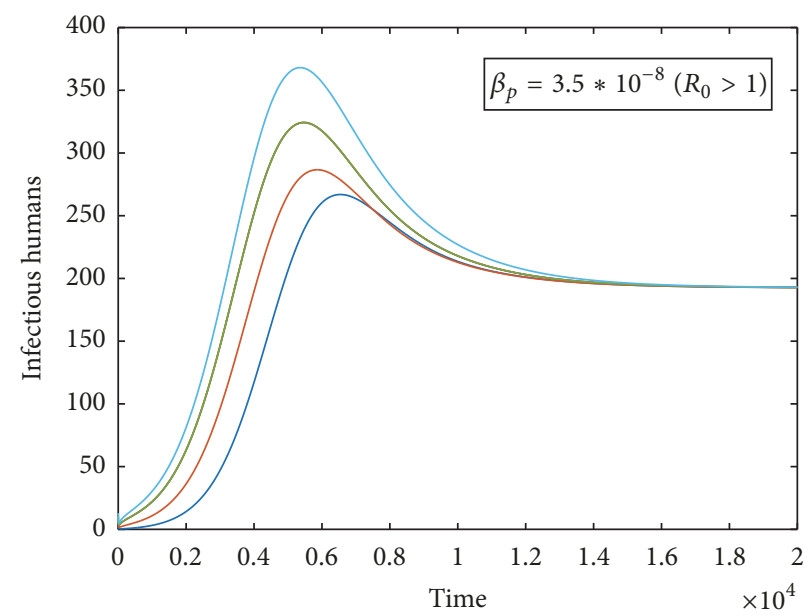

(b)

Figure 1: (a) All solutions of $I_{h}(t)$ converge to the disease-free steady state eventually if $\beta_{p}<\beta_{p}^{*}\left(R_{0}<1\right)$. (b) All solutions of $I_{h}(t)$ converge to the endemic steady state eventually if $\beta_{p}>\beta_{p}^{*}\left(R_{0}>1\right)$.

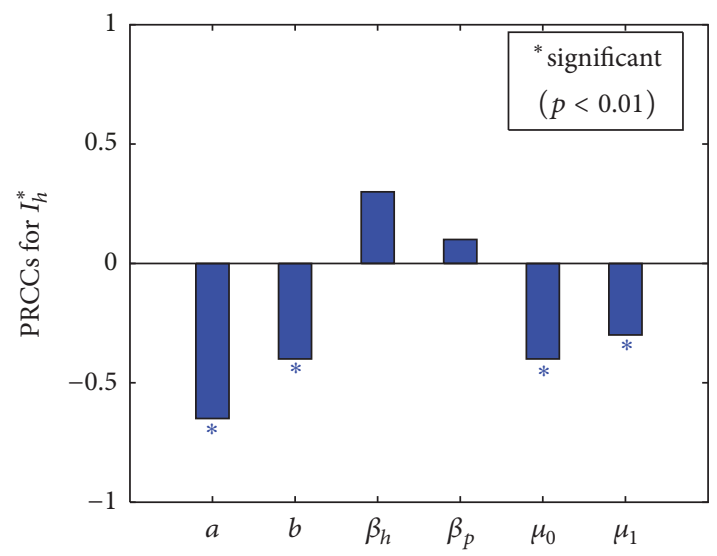

(a)

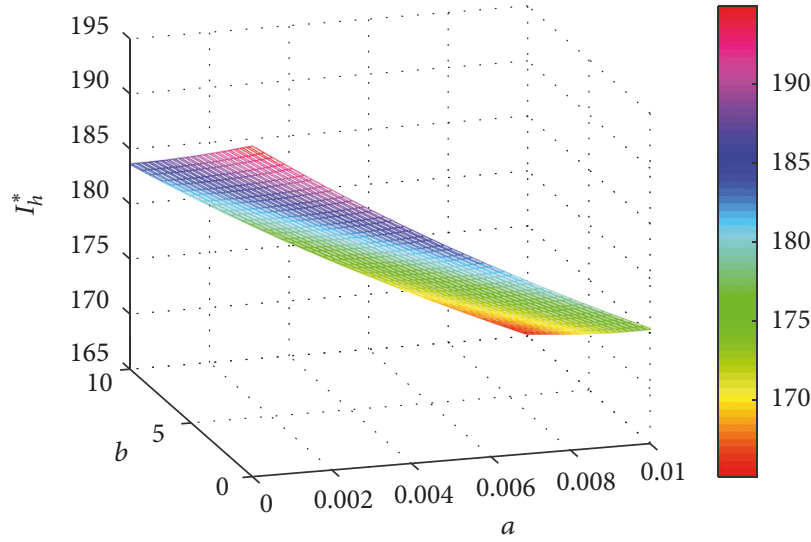

(b)

FIgURE 2: (a) PRCCs for the endemic equilibrium prevalence. All the parameters came from Latin hypercube sampling. (b) Plot of the endemic equilibrium prevalence with respect to the psychological effect parameter $a$ and hospital bed ratio $b$. $\beta_{p}=3.5 * 10^{-8}\left(R_{0}>1\right)$, $\mu_{1}=0.24$.

\section{Conclusions and Discussions}

In this work, in order to evaluate the combined impact of psychological effect and available hospital resources on the transmission of $\mathrm{A}(\mathrm{H} 7 \mathrm{~N} 9)$ virus from poultry to humans, we formulated and analyzed a dynamical model with a nonlinear incidence rate and a nonlinear recovery rate. From the mathematical point of view, we obtained the basic reproduction number $R_{0}$, which determines the extinction of the avian influenza. Theoretical analysis of system (6) indicates that the disease-free equilibrium $E_{02}\left(K_{p}, 0, \bar{S}_{h}, 0,0\right)$ is globally asymptotically stable in $\Gamma$ when the basic reproduction number is less than unity; that is, the avian influenza A(H7N9) will die out (see Figure 1(a)); and the endemic equilibrium $E^{*}\left(S_{p}^{*}, I_{p}^{*}, S_{h}^{*}, E_{h}^{*}, I_{h}^{*}\right)$ is globally asymptotically stable in $\Gamma$ when the basic reproduction number is larger than unity and $b \geq 2 \Lambda\left(\mu_{1}-\mu_{0}-\mu_{h} / 2\right) / \mu_{h}^{2}>0$. Note that although the global stability of endemic equilibrium is obtained under this specific condition, which may be due to the limitations of the analytical method, numerical simulations show that all solutions can converge to $E^{*}$ eventually without the specific condition (see Figure 1(b)).

Both the psychological effect and available hospital resources cannot neither change the stability of endemic equilibrium nor alter the basic reproduction number, but they indeed play a significant role in affecting the number of infectious humans, seen from PRCC results (Figure 2(a)) and the impact of parameters $a$ and $b$ on equilibrium prevalence (Figure 2(b)). Comparing the number of infectious humans with or without psychological effects, that is, parameter $a=0$ or $a>0$, it can be seen that bigger parameter $a$ can significantly decrease the peak of $\mathrm{A}(\mathrm{H} 7 \mathrm{~N} 9)$ infections; meanwhile, the 


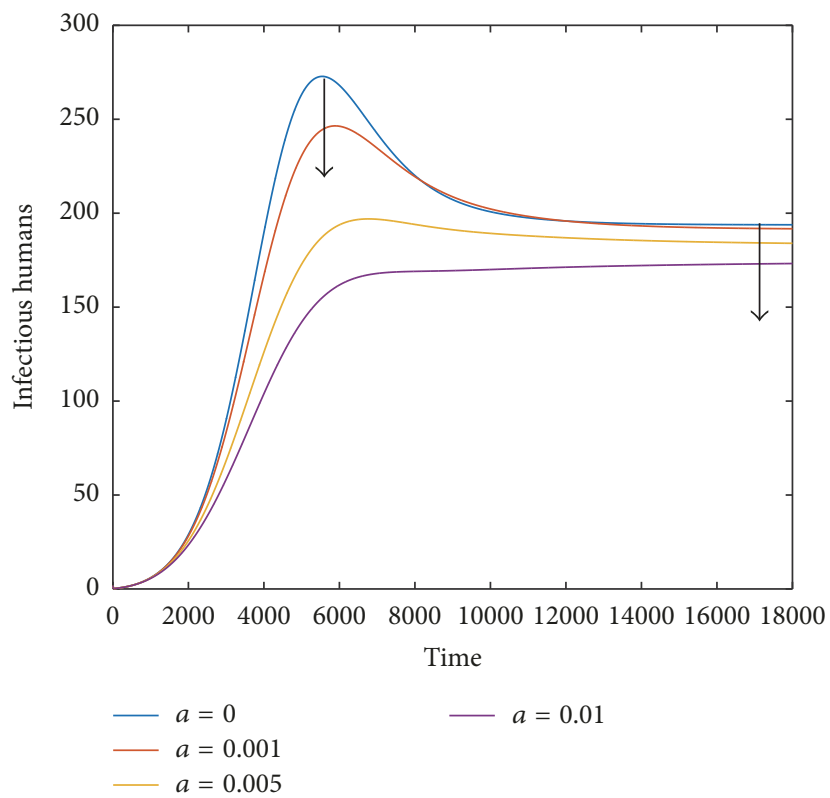

(a)

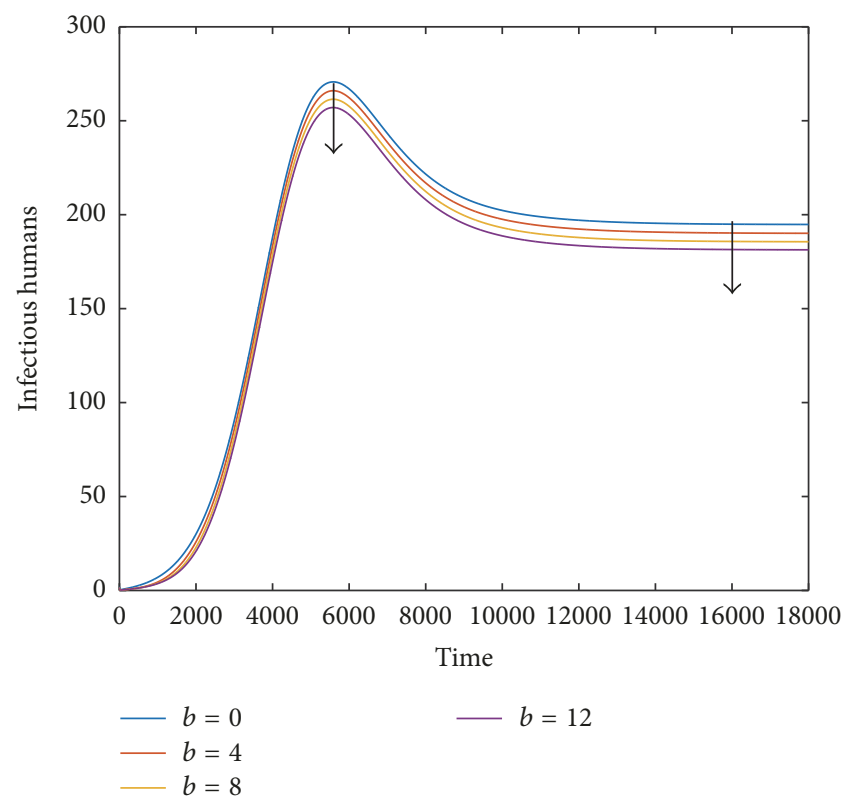

(b)

FIGURE 3: Fix $\beta_{p}=3.5 * 10^{-8}\left(R_{0}>1\right)$. (a) Plot of $I_{h}$ with varying parameter $a$ for $b=1$. (b) Plot of $I_{h}$ with varying parameter $b$ for $a=0.001$. In both cases, the final size and the peak value of the infected are diminished.

final size of the disease can be reduced. However, no matter whether there is psychological effect or not, the disease cannot die out, seen from Figure 3(a). Figure 3(b) indicates that when the available hospital resources are more sufficient, a bigger parameter $b$ leads to a smaller size of the outbreak and a lower number of infectious humans. Similarly, the impact of available hospital resources cannot eradicate the disease either.

Different from the previous avian influenza dynamics models, which usually use bilinear and standard incidence rates and constant recovery rate, in this work, incorporating the combined impact of psychological effect and available hospital resources, we formulate $\mathrm{A}(\mathrm{H} 7 \mathrm{~N} 9)$ dynamic model with nonlinear incidence rate and nonlinear recovery rate. We introduce the recovery function $\mu\left(b, I_{h}\right)=\mu_{0}+b\left(\mu_{1}-\right.$ $\left.\mu_{0}\right) /\left(b+I_{h}\right)$, where parameter $b$ represents hospital bedpopulation ratio, which reflects the available resources of the health care system to public. The number of hospital beds is a critical index and with the number of infected cases increasing it may become a limiting factor in controlling the spread of $\mathrm{A}(\mathrm{H} 7 \mathrm{~N} 9)$ virus. Our results demonstrate that both psychological effect and available hospital resources can dramatically affect the $\mathrm{A}(\mathrm{H} 7 \mathrm{~N} 9)$ virus transmission dynamics. This work is an improvement of existing models of the avian influenza $\mathrm{A}(\mathrm{H} 7 \mathrm{~N} 9)$ and the results can provide some practical implications for the control of $\mathrm{A}(\mathrm{H} 7 \mathrm{~N} 9)$ virus transmission.

Note that, from current data for A(H7N9) infection, there is an incubation period between infection and symptom onset in both avian and human populations [24]. We consider latent class $\left(E_{h}\right)$ in our model, which is more realistic to exhibit the epidemiology of A(H7N9). Based on this characteristic of $\mathrm{A}(\mathrm{H} 7 \mathrm{~N} 9)$ virus, we will incorporate time delay in our model for future study. There have been five seasonal outbreaks of human infection by $\mathrm{A}(\mathrm{H} 7 \mathrm{~N} 9)$ virus in China, since the first outbreak was observed in 2013. Except for the first outbreak, others usually started in October, significantly increased in late December, and then peaked in January of the next year [25]. Thus seasonal variation may affect the spread of $\mathrm{A}(\mathrm{H} 7 \mathrm{~N} 9)$ virus as one of the important factors. Zhao et al. [26] presented a model with period parameters to analyze the effect of climate change on the transmission of A(H7N9) and discussed the global stability and threshold conditions. In our future work, we can also consider the incidence rate as a periodic function.

\section{Conflicts of Interest}

The authors declare that they have no conflicts of interest.

\section{Acknowledgments}

This work is supported by the National Natural Science Foundation of China (NSFC 11401349) and the Foundation for Outstanding Young Scientist in Shandong Province (BS2014SF008).

\section{References}

[1] J. Liu, H. Xiao, Y. Wu et al., "H7N9: A low pathogenic avian influenza A virus infecting humans," Current Opinion in Virology, vol. 5, no. 1, pp. 91-97, 2014. 
[2] R. E. Kahn and J. A. Richt, "The novel H7N9 influenza a virus: Its present impact and indeterminate future," Vector-Borne and Zoonotic Diseases, vol. 13, no. 6, pp. 347-348, 2013.

[3] Study Fingers Chickens, Quail, In Spread Of H7N9 Influenza Virus, 2014, https://www.sciencedaily.com/releases/2014/03/140318093722 .htm.

[4] S. Iwami, Y. Takeuchi, and X. Liu, "Avian-human influenza epidemic model," Mathematical Biosciences, vol. 207, no. 1, pp. $1-25,2007$.

[5] Z. Liu and C.-T. Fang, "A modeling study of human infections with avian influenza A H7N9 virus in mainland China," International Journal of Infectious Diseases, vol. 41, pp. 73-78, 2015.

[6] S. Liu, S. Ruan, and X. Zhang, "Nonlinear dynamics of avian influenza epidemic models," Mathematical Biosciences, vol. 283, pp. 118-135, 2017.

[7] S. Funk, E. Gilad, C. Watkins, and V. A. A. Jansen, "The spread of awareness and its impact on epidemic outbreaks," Proceedings of the National Acadamy of Sciences of the United States of America, vol. 106, no. 16, pp. 6872-6877, 2009.

[8] L. Wang, B. J. Cowling, P. Wu et al., "Human exposure to live poultry and psychological and behavioral responses to influenza A(H7N9), China," Emerging Infectious Diseases, vol. 20, no. 8, pp. 1296-1305, 2014.

[9] P. Wu, L. Wang, B. J. Cowling et al., "Live poultry exposure and public response to influenza $\mathrm{A}(\mathrm{H} 7 \mathrm{~N} 9)$ in urban and rural China during two epidemic waves in 2013-2014," PLoS ONE, vol. 10, no. 9, Article ID e0137831, 2015.

[10] S. Ruan and W. Wang, "Dynamical behavior of an epidemic model with a nonlinear incidence rate," Journal of Differential Equations, vol. 188, no. 1, pp. 135-163, 2003.

[11] S. Liu, L. Pang, S. Ruan, and X. Zhang, "Global dynamics of avian influenza epidemic models with psychological effect," Computational and Mathematical Methods in Medicine, vol. 2015, Article ID 913726, 2015.

[12] R. Liu, J. Wu, and H. Zhu, "Media/psychological impact on multiple outbreaks of emerging infectious diseases," Computational and Mathematical Methods in Medicine, vol. 8, no. 3, pp. 153$164,2007$.

[13] Y. Xiao, T. Zhao, and S. Tang, "Dynamics of an infectious diseases with media/psychology induced non-smooth incidence," Mathematical Biosciences and Engineering, vol. 10, no. 2, pp. 445-461, 2013.

[14] W. Qin, S. Tang, C. Xiang, and Y. Yang, "Effects of limited medical resource on a Filippov infectious disease model induced by selection pressure," Applied Mathematics and Computation, vol. 283, pp. 339-354, 2016.

[15] WHO, Dengue and dengue naemorrhagic fever, Fact Sheet 117, 2012.

[16] A. Abdelrazec, J. Bélair, C. Shan, and H. Zhu, "Modeling the spread and control of dengue with limited public health resources," Mathematical Biosciences, vol. 271, pp. 136-145, 2016.

[17] W. M. Liu, S. A. Levin, and Y. Iwasa, "Influence of nonlinear incidence rates upon the behavior of SIRS epidemiological models," Journal of Mathematical Biology, vol. 23, no. 2, pp. 187204, 1986.

[18] C. Shan and H. Zhu, "Bifurcations and complex dynamics of an SIR model with the impact of the number of hospital beds," Journal of Differential Equations, vol. 257, no. 5, pp. 1662-1688, 2014.
[19] O. Diekmann, J. A. P. Heesterbeek, and M. G. Roberts, "The construction of next-generation matrices for compartmental epidemic models," Journal of the Royal Society Interface, vol. 7, no. 47, pp. 873-885, 2010.

[20] P. van den Driessche and J. Watmough, "Reproduction numbers and sub-threshold endemic equilibria for compartmental models of disease transmission," Mathematical Biosciences, vol. 180, pp. 29-48, 2002.

[21] J. Lucchetti, M. Roy, and M. Martcheva, "An avian influenza model and its fit to human avian influenza cases," Advances in Disease Epidemiology, pp. 1-30, 2009.

[22] S. M. Blower and H. Dowlatabadi, "Sensitivity and uncertainty analysis of complex models of disease transmission: an HIV model, as an example," International Statistical Review, vol. 62, no. 2, pp. 229-243, 1994.

[23] S. Marino, I. B. Hogue, C. J. Ray, and D. E. Kirschner, "A methodology for performing global uncertainty and sensitivity analysis in systems biology," Journal of Theoretical Biology, vol. 254, no. 1, pp. 178-196, 2008.

[24] S. Liu, S. Ruan, and X. Zhang, "On avian influenza epidemic models with time delay," Theory in Biosciences, vol. 134, no. 3-4, pp. 75-82, 2015.

[25] L. Zhou, R. Ren, L. Yang et al., "Sudden increase in human infection with avian influenza A(H7N9) virus in China, SeptemberDecember 2016," Western Pacific surveillance and response journal: WPSAR, vol. 8, no. 1, pp. 6-14, 2017.

[26] X.-Y. Zhao, S.-M. Guo, M. Ghosh, and X.-Z. Li, "Stability and persistence of an avian influenza epidemic model with impacts of climate change," Discrete Dynamics in Nature and Society, vol. 2016, Article ID 7871251, 2016.

[27] Y. Xiao, X. Sun, S. Tang, and J. Wu, "Transmission potential of the novel avian influenza A(H7N9) infection in mainland China," Journal of Theoretical Biology, vol. 352, pp. 1-5, 2014. 


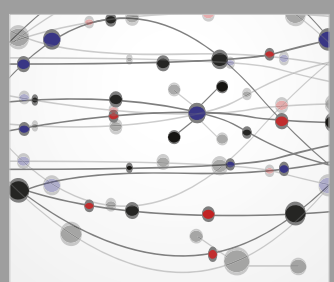

The Scientific World Journal
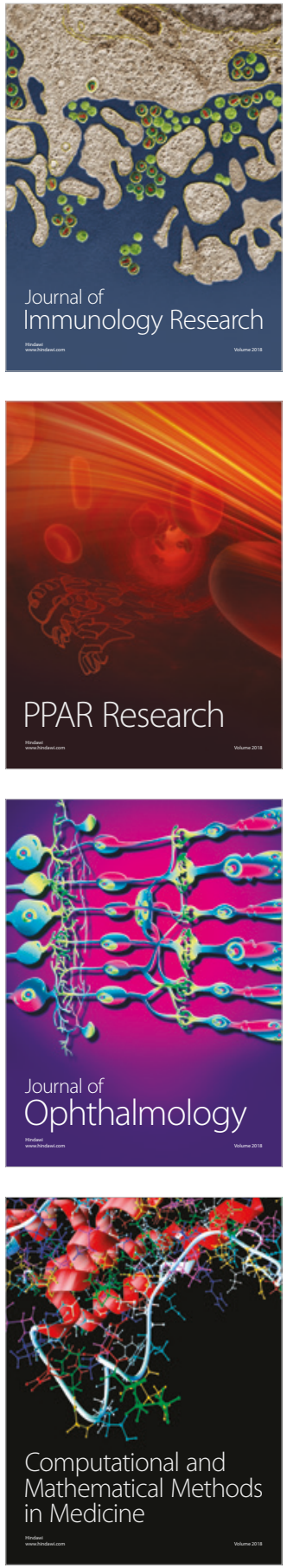

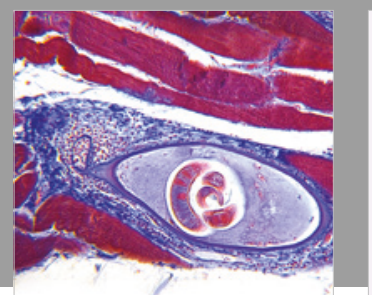

Gastroenterology Research and Practice

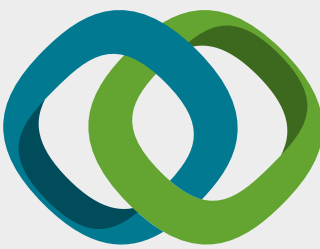

\section{Hindawi}

Submit your manuscripts at

www.hindawi.com
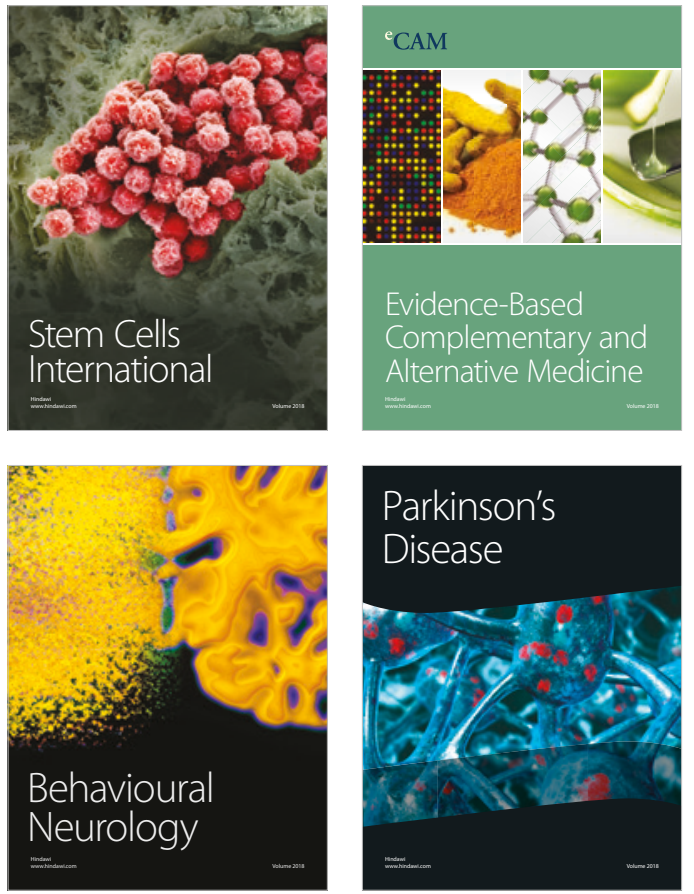

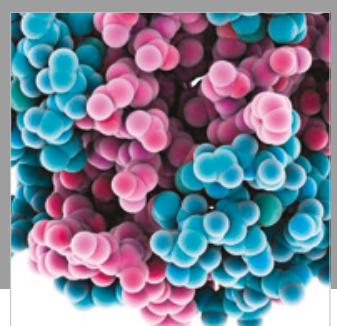

ournal of

Diabetes Research

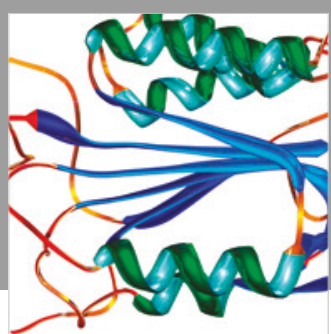

Disease Markers
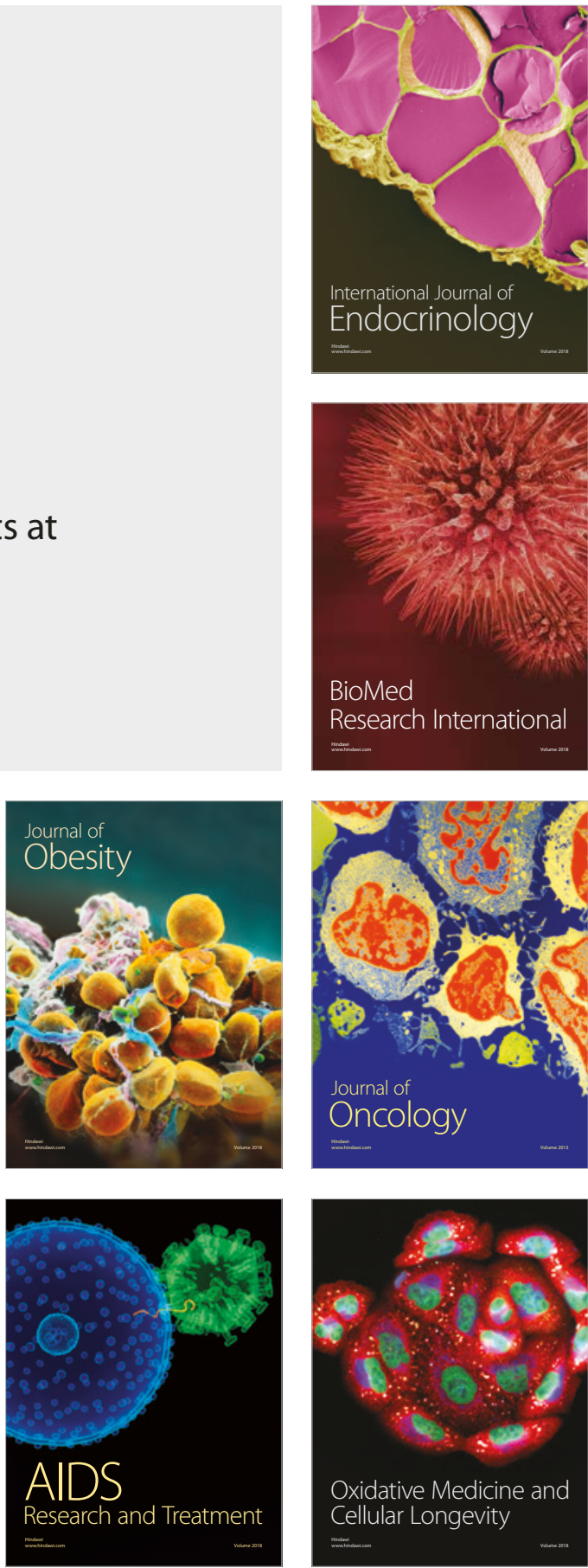